\title{
To Compare The Efficacy Of Titanium Versus Teflon Piston On Hearing Outcome In Terms Of Air Conduction Gain And Air Bone Gap Closure In LASER Stapedotomy - A Retrospective Study
}

\author{
Authors: Swati Suneha ${ }^{17}$, Kranti Bhavana $^{2}$, Ragini Raina ${ }^{1}$, Meenu Kalyani ${ }^{3}$, Bhartendu Bharti ${ }^{4}$ \\ Authors Affiliations: (1) Senior Resident, (2) Additional Professor \& Head of Department, (3) Audiologist and \\ speech-language pathologist, (4) Associate Professor, \\ Department of Otorhinolaryngology-Head and Neck Surgery, All India Institute of Medical Sciences, Patna
}

\begin{abstract}
:
Aim: To determine the efficacy of Titanium versus Teflon piston on hearing outcome in terms of Air Conduction Threshold (ACT) gain and Air Bone Gap $(A B G)$ closure in patients undergoing LASER stapedotomy.
\end{abstract}

\begin{abstract}
Methods: This retrospective study was conducted at the department of ENT and Head and Neck Surgery, AlIMS Patna. 40 patients underwent LASER stapedotomy. Both Teflon $(0.4 \mathrm{~mm}$ and $0.6 \mathrm{~mm} \mathrm{D}$, Grace Medical Devices) and Titanium (0.4mm D, Kurz Medical Devices) pistons were inserted randomly. Crimping of titanium Kurz piston was not required due to its unique design which fits on to the incus long process like a clip whereas all the Teflon pistons required crimping.Titanium (Kurz Medical Devices) pistons are costlier in comparison to Teflon (Grace Medical Devices) pistons. Preoperative and 3 monthly postoperative Pure Tone Audiograms (PTA) wereobtained. ACT (Air conduction threshold) gain and ABG (Air Bone Gap) closure were measured.Data was analyzed with IBM SPSS Statistics software Version 20. Descriptive analysis, paired and unpaired t-tests were applied. P-value of $<0.05$ at $95 \%$ confidence levels was considered statistically significant.
\end{abstract}

Results: 40 patients (M: F: 3:7) underwent LASER stapedotomy.Titanium piston was utilized in 11 and Teflon in 29 patients. Patients with titanium piston experienced a mean ACT gain of $21.51 \pm 05.42 \mathrm{dBand}$ mean ABG closure of $12.27 \pm 05.18 \mathrm{~dB}$. Those with Teflon piston gained a meanACT gain of $18.54 \pm 04.43$ $\mathrm{dB}$ and mean $\mathrm{ABG}$ closure of $11.96 \pm 06.12 \mathrm{~dB}$.Both groups experienced statistically significant hearing gain $(p<0.05)$. Both groups were however comparable and the difference between these parameters was statistically insignificant $(p>0.05)$.Conclusion: Both titanium and Teflon pistons are equally efficacious in improving postoperative hearing gain in LASER stapedotomy patients in terms of $A C T$ gain and $A B G$ closure. Titanium Kurz pistons do not require crimping making it easier to use in comparison to the Teflon pistons which needs to be crimped every time though cost may be a limiting factor in the usage of Titanium pistons.Both types of pistons can be satisfactorily employed in this surgical procedure if we consider the hearing gain post operatively.

Study design: A retrospective observational study

Keywords: Otosclerosis, Teflon, Titanium, Air Conduction Threshold (ACT), Air Bone Gap (ABG) closure

\section{Introduction:}

Otosclerosis is characterized by abnormal bone remodeling bony labyrinth; resulting in progressive bilateral conductive hearing loss, often accompanied by tinnitus. 1 Initially there is conductive hearing impairment and the sensorineural component arises on the involvement of the spiral ligament in cochlear otosclerosis.2.3Abnormal bone remodeling is seen in genetically and environmentally predisposed patients, resulting in stapes fixation and conductive hearing loss. This disease follows an autosomal dominant inheritance pattern with incomplete penetrance.4,50ther factors like measles infection and hormonal stress have also been implicated with raised frequency or severity of the disease, though various studies are still inconclusive.6,7 Pure cochlear endosteum involvement leads to sensorineural loss. However, these cases usually are severe enough to have varying degrees of stapes footplate involvement, causing a mixed hearing loss pattern.4,8-9Around $65-90 \%$ of patients experience intermittent or continuous tinnitus.10-13 Vestibular manifestations due to biochemical changes in 
perilymph include positional or spontaneous dizziness. This may or may not be recurrent.14-16

Diagnosis is based on both clinical findings and audiological evaluation. These patients present with an intact tympanic membrane and no evidence of eustachian tube dysfunction on examination. Once suspected of otosclerosis, these undergo detailed audiological evaluation. Conductive hearing loss, with elevated bone conduction thresholds which peaks at $2 \mathrm{kHz}$ is typical of the disease. 3 An'As'type of tympanogram on impedance audiometry is suggestive of reduced middle ear ossicular mobility due to stapes footplate fixation. A high-resolution CT imaging showing pericochlear hypodense double ring sign assists in diagnosis, as well as preoperative evaluation of middle and inner ear anatomy, with about $90 \%$ sensitivity. 18

So far, the best and safe treatment option has been a surgical intervention in the form of a stapedectomy or stapedotomy. Stapedotomy is preferred with fenestration being performed with microdrill, handheld perforator or LASER.19LASER stapedotomy has given better results in terms of less footplate mobilization and inner ear mechanical trauma, better air-bone gap closure rates, and rarer incidences of sensorineural hearing loss; thus becoming popular worldwide for both primary and revision stapedotomies.20-23

A wide range of factors governs the surgical outcome. The type and diameter of the piston, preoperative good bone conduction thresholds with at least $20 \mathrm{~dB}$ air-bone gap, type of Otosclerosis with the extent of footplate involvement, surgical technique (partial Stapedectomy, total Stapedectomy, Stapedotomy), and their modifications and tissue sealant around prosthesis are some of them being studied.24,25

Owing to relentless research and innovation, we have at our disposal a range of prostheses of different design, material, diameter, weight and modifications to allow incus anchoring. Materials like steel, platinum, gold, Teflon, titanium, and alloys like nitinol have been utilized for prosthesis making, each with their own clientele. While Teflon piston is the most commonly employed, titanium piston has been recently developed and steadily gaining popularity among surgeons. Larger piston diameter has been shown to result in a better hearing gain which is more pronounced at lower frequencies. Generally, both titanium and Teflon pistons have given good hearing results over time, and hence are being routinely utilized in stapedotomies.19

The objective of our study was to determine the efficacy of Titanium versus Teflon piston on hearing outcomes in terms of ABG closure in patients undergoing LASER Stapedotomy and to observe for postoperative subjective symptom improvement and complications.

\section{Materials and methods:}

It was a retrospective, non-randomized, nonblindedstudy of 40 patients at the Department of ENT, Head and Neck Surgery, AIIMS Patna from February 2018 to December 2019. Patients with a clinical and audiometric diagnosis of otosclerosis underwent LASER stapedotomy with prosthesis insertion. The operating team was the same for all the patients. Patients were evaluated with Pure Tone Audiometry (PTA) and impedance audiometry to ascertain the diagnosis. Patients were counseled about the disease etiopathology, progression, and various available treatment modalities, including wait and watch, sodium fluoride administration, and exploratorytympanotomy. Once planned for surgery, surgical fitness was determined preoperatively.A high-resolution $\mathrm{CT}$ imaging was obtained to look for possible radiologic evidence of otosclerosisand to evaluate ossicular erosion/deformity, squamous disease, and facial nerve status.

Both Teflon $(0.4 \mathrm{~mm}$ and $0.6 \mathrm{~mm} \mathrm{D,} \mathrm{Grace} \mathrm{Medical}$ Devices) and Titanium $(0.4 \mathrm{~mm} D$, Kurz Medical Devices) were used as prosthesis (figures 1 and 2). The selection of prosthesis type was non-randomized and choice of the prosthesis was given to the patients after relevant counseling. Patients were explained the expected hearing outcomes and the costeffectiveness of the piston types. Informed consent was taken for the surgery and its probable outcomes and complications.Kurz titanium piston was chosen by the operating surgeon due to its ease of application. It needed no crimping and could be clipped to the long process of incus without much manipulation of the ossicles. Though titanium pistons come at higher cost in comparison to Teflon pistons but its ease of application without any need for crimping made it a good piston to use.

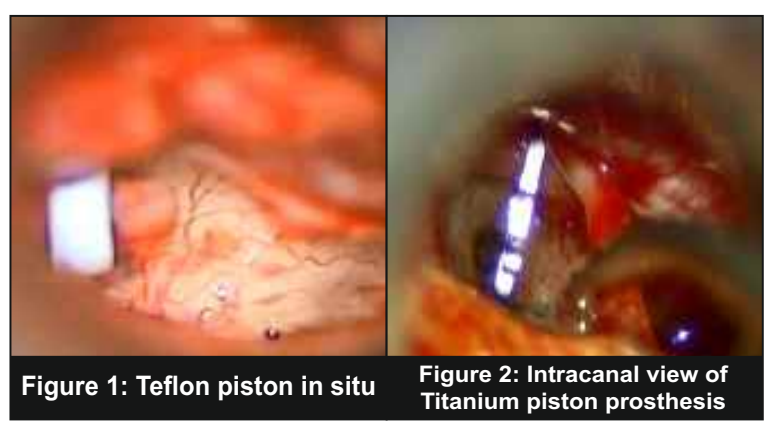


All patients underwent LASER stapedotomy under general anaesthesia. A transcanal approach was made to access the middle ear. The tympanomeatal flap was elevated, middle ear ossicles inspected and stapes footplate fixity ascertained. Stapedius tendon was released and the posterior crus cut with straight free beam CO2 LASER. After incudo-stapedial joint dislocation with a curved pick, the stapes suprastructure was out-fractured and was removed. The distance from the incus to the footplate was measured for adjusting the length of the prosthesis. Stapedotomy was done with a single shot circular CO2 LASER beam. Kurz titanium pistons once placed in the fenestra did not require crimping as it can be easily "clipped" to the incus long process. On the other hand, all Teflon pistons required crimping. After placement and crimping of the prosthesis to incus long process, a fat graft was used to stabilize it around the footplate. The tympanomeatal flap was repositioned.

All patients received postoperative steroids and antibiotics for one week. They were followed up on an outpatient basis for relevant oto-endoscopic inspection and audiological investigations.

Pure Tone Audiometry were performed at 1 month and 3 months postoperative intervals.Air Conduction Threshold (ACT) at frequencies of $0.25,0.5,1,2,4$, and $8 \mathrm{kHz}$ and Bone Conduction Threshold (BC) at 0.5, 1, 2, and $4 \mathrm{kHz}$ were recorded and Air Bone Gap (ABG) was calculated. ACT gain and ABG closure were taken as measures of hearing improvement, as per recommended guidelines.Subjective symptom improvement in terms of reduced or cured tinnitus, dizziness, and overall improved quality of life was also assessed.Audiological procedures were conducted with a calibrated clinical audiometer (MAICO NA 42).

Data was analyzed with IBM SPSS Statistics software Version 20. Descriptive analysis,paired and unpaired t-tests were utilized. $P$ value was calculated at $95 \%$ confidence levels and value of $<0.05$ was taken as statistically significant. Both Teflon and titanium groups werecompared in terms of the amount of ACT gain, AB gap closure, and results were analyzed.

\section{Results:}

Our data revealed that the mean age of patients at intervention was $29.87 \pm 9.57$ years(M: F:3:7). The mean age of patients with Teflon piston was $28.34 \pm 7.76$ years and that of those with titanium piston was $33.91 \pm 1.82$ years, which was comparable. A total of $29(72.5 \%)$ patients chose Teflon piston while $11(27.5 \%)$ patients opted for titanium prosthesis. Table 1 details the relevant demographic data.

Table 1: Patient demographic data

\begin{tabular}{|c|c|c|}
\hline $\begin{array}{l}\text { Patient } \\
\text { demographics }\end{array}$ & Frequency & $\begin{array}{l}\text { Percentage } \\
(\%)\end{array}$ \\
\hline \multicolumn{3}{|l|}{ Age group (years) } \\
\hline $10-20$ & 07 & 17.5 \\
\hline $21-30$ & 04 & 10.0 \\
\hline $31-40$ & 23 & 57.5 \\
\hline $41-50$ & 04 & 10.0 \\
\hline $51-60$ & 02 & 05.0 \\
\hline Total & 40 & 100 \\
\hline Mean age (years) & 29.87 & \\
\hline \multicolumn{3}{|l|}{ Gender } \\
\hline Male & 13 & 32.5 \\
\hline Female & 27 & 67.5 \\
\hline \multicolumn{3}{|l|}{ Laterality } \\
\hline Right & 23 & 57.5 \\
\hline Left & 17 & 42.5 \\
\hline \multicolumn{3}{|l|}{ Piston } \\
\hline Teflon & 29 & 72.5 \\
\hline Titanium & 11 & 27.5 \\
\hline
\end{tabular}

Table 2 details the relevant audiological outcomes. Patients in Teflon group achieved a mean gain of $18.54 \mathrm{~dB}$ (SD $4.43 \mathrm{~dB}$, SE of mean=0.82, $\mathrm{t}=22.55, \mathrm{df}=28$ ) in $\mathrm{ACT}$ levels while those in Titanium group gained a mean21.51 dB (SD $5.42 \mathrm{~dB}$, SE of mean=1.63, $\mathrm{t}=13.16, \mathrm{df}=10$ ). While both groups experienced a significant gain in the ACT $(p<0.05)$, the difference between these two groups was statistically insignificant ( $p=0.0831, t=1.78, d f=38$ ). Similarly, Titanium group had an $A B G$ closure of $12.27 \mathrm{~dB}$ (SD $5.18 \mathrm{~dB}$, SE of mean 1.56, $\mathrm{t}=7.86, \mathrm{df}=10$ ) and Teflon group had that of $11.96 \mathrm{~dB}$ (SD $6.12 \mathrm{~dB}, \mathrm{SE}$ of mean 1.14, $t=10.54, d f=28)$. While these closure rates were significant on their own $(p<0.05)$, the difference between them was not so $(p=0.8863$, $\mathrm{t}=0.14, \mathrm{df}=38$ ).

Table 2: Mean PTA improvements and ABG closure rates

\begin{tabular}{|l|lc|l|}
\hline $\begin{array}{l}\text { Mean } \\
\text { hearing } \\
\text { thresholds } \\
\text { (dB) }\end{array}$ & Teflon & Titanium \\
\hline Preop ACT & $\begin{array}{l}58.80 \\
12.28\end{array}$ & \pm & $62.60 \pm 11.82$ \\
\hline Postop ACT & \begin{tabular}{llll|}
40.26 \\
12.87
\end{tabular} & \pm & $41.09 \pm 16.01$ \\
\hline ACT Gain & 18.54 & \pm & $21.51 \pm 05.42$ \\
& 04.43 & & \\
\hline Preop ABG & 20.34 & \pm & $20.91 \pm 05.39$ \\
& 06.94 & & \\
\hline Postop ABG & 08.38 & \pm & $08.64 \pm 03.23$ \\
& 03.29 & & \\
\hline ABG & 11.96 & \pm & $12.27 \pm 05.18$ \\
Closure & 06.12 & & \\
\hline
\end{tabular}


Hence both Titanium and Teflon pistons gave comparable results and were both found to be equally efficacious in providing good surgical and audiological outcomes (Figure 3). None of our patients experienced debilitating complications like facial palsy, worsened tinnitus,or vestibular disturbances. All reported subjective improvement in symptom profile post-surgery.

Figure 3: Comparison of $A C T$ gain and $A B G$ closure among both groups.

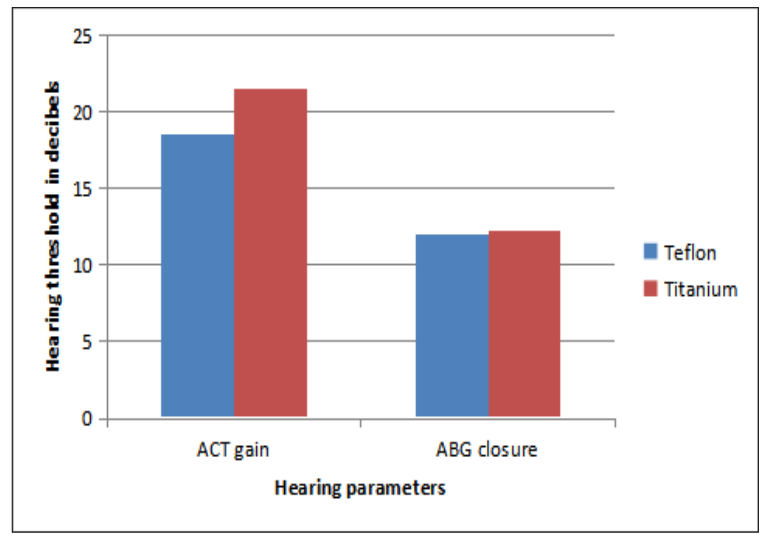

\section{Discussion:}

Numerous studies have been conducted over time comparing audiological results of different types of prosthesis. The data analysis is complicated due to various factors affecting the final outcomelike piston material,surgeon's experience and technique, fenestration size, stapedius tendon status, disease severity, prosthesis design, andrevision surgery.190verall, stapedotomy with prosthesis insertion has given consistent results over time and has proven to be a better modality than complete or partial stapedectomy, given lesser intraoperative and postoperative complications.27LASER Stapedotomy is a promising technique with a safer complication spectrum and hence has been employed at our center exclusively.20,21

This study population had a comparable age distribution among both Teflon and titanium groups. The mean age of our patients was 29.87 years. This was similar to the study conducted by Singh and Goyal where the mean age of patients was 29.1 years. 27 Males formed $32.5 \%$ of the patient population whereas females were $67.5 \%$. Though being a disease with a female preponderance, some studies have also found this to be a less predominant feature.26,27 The laterality of operated ear in this study (right-57.5\%, left-42.5\%) was similar to that found by Alzhrani F et al. (right-58.5\%, left-41.5\%).26 Pistons of both $0.4 \mathrm{~mm}$ and $0.6 \mathrm{~mm}$ thickness have been utilized at different centers, with $0.6 \mathrm{~mm}$ preferred by many authors. Mangham et al compared $0.6 \mathrm{~mm}$ Teflon and titanium pistons and concluded that for the given actual diameter, both gave similar results. However, since the actual Teflon diameter was about $10 \%$ larger, it gave better ABG closure when corrected for the size data.19. In this study Teflon pistons of both $0.4 \mathrm{~mm}$ and $0.6 \mathrm{~mm}$, and a titanium piston of $0.4 \mathrm{~mm}$ diameter were used.

Improvements in $A C T$ and $A B G$ closure are considered to be reliable indicators of efficacy of Stapedotomy. In this study both groups of patients experienced statistically significant improvement in postoperative ACT gain and ABG closure, thus reaffirming the efficacy of LASER Stapedotomy in Otosclerosis management. This study also concluded that both titanium and Teflon pistons gave almost identical hearing results in terms of the abovementioned parameters. Hence there is no statistically significant advantage of using one piston over the other and both are equally efficacious.These results are in harmony with other studies that found comparable results when using different types of prostheses. The main advantage of a clip piston was its ease of application as it needed no crimping. Cost was a limiting factor for usage of titanium pistons as it was costlier than the Teflon pistons. Bansal M. concluded both titanium and Teflon pistons to be equally effective; Tange et al.found both crimping and non-crimping titanium prostheses to give statistically similar results; Casale et al. inferred both fluoroplastic Teflon and titanium pistons to be equally good in providing adequate hearing gain.19This study is limited by its non-randomized nature, resulting in the sample size of each group being different. A prospective study with randomization of piston insertion would help to better compare both groups of patients and may discern any possible difference in hearing outcomes.

\section{Conclusion:}

LASER stapedotomy with prosthesis insertion is the current standard treatment for otosclerosis management and provides clinically and statistically significant hearing improvement in patients. We currently have an array of prosthesis materials at our disposal today, and all of these often provide similar results in hearing gain. Both titanium and Teflon pistons give comparable results and thus can be effectively employed in stapedotomies as per the surgeon's and patient's preference and availability of these. Clip pistons have advantage over Teflon in terms of its non-crimping nature which helps in minimizing ossicular movement and ensures better 
fit to the long process of incus. Its unique design avoids lenticular necrosis and subsequent failures due to displacement of prosthesis. Cost is a limiting factor in our set up.More studies with a larger sample size are however required to form clear consensus and guidelines regarding these prostheses.

Funding: No funding sources.

\section{Conflict of interest: None.}

Compliance with ethical standards: All procedures contributing to this study have been conducted complying with the Helsinki Declaration of 1975 institutional guidelines.

\section{Reference:}

1. Spear SA, Crawford JV. Comparison of nitinol stapes pistons with conventional stapes pistons: a cadaver study. ISRN Otolaryngol. 2011;2011:932849.

2. Antoli-Candela F Jr McGill T, Peron D. Ann OtolRhinolLaryngol.Histopathological observations on the cochlear changes in otosclerosis. 1977 Nov-Dec;86(6 Pt 1):81320.

3. Cureoglu S, Baylan MY, Paparella MM. Cochlear otosclerosis. CurrOpinOtolaryngol Head Neck Surg. 2010;18:357-62.

4. Schuknecht HF, Kirchner JC. Cochlear otosclerosis: fact or fantasy. Laryngoscope. 1974;84:766-782.

5. Brownstein Z, Goldfarb A, Levi $\mathrm{H}$, et al. Chromosomal mapping and phenotypic characterization of hereditary otosclerosis linked to the OTSC4 locus. Arch Otolaryngol Head Neck Surg. 2006;132:416-424.

6. Arnold W, Niedermeyer HP, Lehn N, et al. Measles virus in otosclerosis and the specific immune response of the inner ear. Acta Otolaryngol. 1996;116:705-709.

7. Markou K, Goudakos J. An overview of the etiology of otosclerosis. Eur Arch Otorhinolaryngol. 2009;266:25-35.

8. Frisch T, Sorensen MS, Overgaard S, Bretlau P. Estimation of volume referent bone turnover in the otic capsule after sequ-ential point l a b e I ing. A n n o tol RhinolLaryngol. 2000;109:33-39.

9. McKenna MJ, Kristiansen AG. Molecular biology of otosclerosis. Adv Otorhinolaryngol. 2007;65:68-74.
10. Rajati $M$, Poursadegh $M$, Bakhshaee $M$, Abbasi A, Shahabi A. Outcome of stapes surgery for tinnitus recovery in otosclerosis. Int Tinnitus J. 2012;17:42-6.

11. Bast F, Mazurek B, Schrom T. Effect of stapedotomy on pre-operative tinnitus and its psychosomatic burden. Auris Nasus Larynx. 2013;40:530-3.

12. Ismi O, Erdogan O, Yesilova M, Ozcan C, Ovla D, Gorur K. Does stapes surgery improve tinnitus in patients with otosclerosis? Braz J Otorhinolaryngol. 2017;83:568-73. SkarżyńskiH

13. Gos E, Dziendziel B, Raj-Koziak D, Włodarczyk EA, Skarżyński PH. Clinically important change in tinnitus sensation after stapedotomy. Health Qual Life Outcomes. 2018 Nov 6;16(1):208.

14. Sando I, Hemenway WG, Miller DR, et al. Vestibular pathology in otosclerosis temporal bone histopathological report. Laryngoscope. 1974;84:593-605.

15. Lawrence M. Possible influence of cochlear otosclerosis on inner ear fluids. Ann Otol. 1966;75:553-558.

16. Paparella MM, Mancini F, Liston SL. Otosclerosis and Meniere's syndrome: diagnosis and treatment. Laryngoscope. 1984;94:1414-1417.

17. Carhart R. Clinical applications of bone conduction audiometry. Arch Otolaryngol. 1950;51:798-808.

18. Quesnel AM, Moonis G, Appel J, et al. Correlation of computed tomography with histopathology in otosclerosis. OtolNeurotol.2013;34(1):22-28.

19. Bansal M. Teflon Implants Versus Titanium Implants in Stapes Surgery. Indian J Otolaryngol Head Neck Surg. 2016;68(1):16-19.

20. Jovanovic S, Scho" nfeld U, Scherer H. CO2 laser stapedotomy with the "oneshot" technique - clinical results. Otolaryngol Head Neck Surg2004;131:750-7.

21. Cuda D, Murri A, Mochi P, Solenghi T, Tinelli N. Microdrill, CO2-laser, and piezoelectric stapedotomy: a comparative study. OtolNeurotol2009;30:1111-5.

22. Lesinski SG, Stein JA. Stapedectomy revision 
with the CO2 laser. Laryngoscope 1989;99:13-9.

23. Häusler R, Messerli A, Romano V, Burkhalter $\mathrm{R}$, Weber HP, Altermatt HJ. Experimental and clinical results of fiberoptic argon laser $\mathrm{s} t \mathrm{a} \mathrm{p}$ e d o to m y. E u r A r h Otorhinolaryngol. 1996;253:193-200.

24. Shah V, Ganapathy H. Factors Affecting the Outcome of Stapes Surgery. Indian J Otolaryngol Head Neck Surg. 2018;70(2):256-261.

25. Quaranta N, Piccininni K, Romanello $M$, Lucidi D, Sergi B. The impact of intraoperative factors in otosclerosis outcomes: retrospective study in a tertiary centre. Acta Otorhinolaryngol Ital. 2019;39(3):197-204.

26. Alzhrani F, Mokhatrish MM, Al-Momani MO, Alshehri H, Hagr A, Garadat SN. Effectiveness of stapedotomy in improving hearing sensitivity for 53 otosclerotic patients: retrospective review. Ann Saudi Med. 2017;37(1):49-55.

27. Singh PP, Goyal A. Our experience with the $\mathrm{t}$ it a n i u m sof $\mathrm{t}$ clip piston stapedotomy. Indian J Otolaryngol Head Neck Surg. 2013;65(3):280-282.

\section{Address for correspondence:}

Dr. Swati Suneha

Senior Resident

Department of Otorhinolaryngology-Head and Neck Surgery,

All India Institute of Medical Sciences, Phulwari sharif Patna-801507, Bihar, India

Email-sunehaswati@gmail.com

Telephone-91-8825304721 